\title{
Integrated motivational interviewing and cognitive behaviour therapy for lifestyle mediators of overweight and obesity in community-dwelling adults: a systematic review and meta-analyses
}

\author{
Stephen Barrett ${ }^{1}$, Stephen Begg ${ }^{1}$, Paul O'Halloran ${ }^{2}$ and Michael Kingsley ${ }^{1 *}$
}

\begin{abstract}
Background: The aim of this study was to investigate whether integrated motivational interviewing and cognitive behaviour therapy leads to changes in lifestyle mediators of overweight and obesity in community-dwelling adults.

Method: Six electronic databases were systematically searched up to 04 October, 2017. Analyses were restricted to randomised controlled trials that examined the effect of integrated motivational interviewing and cognitive behaviour therapy on lifestyle mediators of overweight and obesity (physical activity, diet, body composition) in community-dwelling adults. Meta-analyses were conducted using change scores from baseline in outcome measures specific to the lifestyle mediators of overweight and obesity to determine standardized mean differences (SMD) and 95\% confidence intervals (95\% Cl). The Grades of Recommendation, Assessment, Development and Evaluation approach was used to evaluate the quality of the evidence.

Results: Ten randomised controlled trials involving 1949 participants were included. Results revealed moderate quality evidence that integrated motivational interviewing and cognitive behaviour therapy had a significant effect in increasing physical activity levels in community-dwelling adults (SMD: 0.18, 95\% Cl: 0.06 to 0.31 , $p<0.05)$. The combined intervention resulted in a small, non-significant effect in body composition changes (SMD: $-0.12,95 \% \mathrm{Cl}:-0.24$ to $0.01, p=0.07$ ). Insufficient evidence existed for outcome measures relating to dietary change.

Discussion: The addition of integrated motivational interviewing and cognitive behaviour therapy to usual care can lead to modest improvements in physical activity and body composition for community-dwelling adults. The available evidence demonstrates that it is feasible to integrate MI with CBT and that this combined intervention has the potential to improve health-related outcomes.
\end{abstract}

Conclusion: This review details recommendations for future research including the adoption of uniform objective outcome measures and well-defined interventions with sufficient follow-up durations and assessments of treatment fidelity.

Keywords: Health promotion, Health behaviour, Obesity, Physical activity, Body composition

\footnotetext{
* Correspondence: M.Kingsley@latrobe.edu.au

'La Trobe University, La Trobe Rural Health School, PO Box 199, Bendigo, VIC

3552, Australia

Full list of author information is available at the end of the article
}

(c) The Author(s). 2018 Open Access This article is distributed under the terms of the Creative Commons Attribution 4.0 International License (http://creativecommons.org/licenses/by/4.0/), which permits unrestricted use, distribution, and reproduction in any medium, provided you give appropriate credit to the original author(s) and the source, provide a link to the Creative Commons license, and indicate if changes were made. The Creative Commons Public Domain Dedication waiver (http://creativecommons.org/publicdomain/zero/1.0/) applies to the data made available in this article, unless otherwise stated. 


\section{Background}

The epidemic of overweight and obesity continues to rise worldwide, and constitutes a serious public health concern [1]. In 2016, more than 650 million adults were classified as obese (according to the World Health Organizations [WHO] body mass index [BMI] classification of obese $\geq$ $30 \mathrm{~kg} / \mathrm{m}^{2}$ ) [2]. Overweight and obesity presents a major challenge to population health due to its intricate association with a number of chronic diseases [3]. Overweight and obese individuals experience increased morbidity, functional limitations and psychosocial problems as a result of excess adiposity [4]. Due to the increasing prevalence of overweight and obesity, developing effective treatment approaches has been identified as a research and population health priority [5].

The aetiology of obesity is complex and multifaceted [6]. However, as individual and personal choices play a critical role in the manifestation of overweight and obesity, behaviour modification and lifestyle interventions are recommended as the primary steps in overweight and obesity management $[7,8]$. Interventions typically target changes in lifestyle mediators of overweight and obesity namely physical activity (PA), diet, and body composition (waist circumference; mass; body mass index) [7, 9]. Psychological strategies such as increasing motivation for change, improving self-efficacy, and self-regulatory capabilities are required for addressing the lifestyle mediators of overweight and obesity and are the best predictors of beneficial physical activity and weight outcomes [8, 10-12].

Motivational interviewing (MI) is a directive, behaviour change technique that is effective in overcoming ambivalence and increasing desire for behaviour change [13]. The principles and methods of MI address issues associated with ambivalence about behaviour change, including decreased confidence and low self-efficacy [14]. MI has well established efficacy for initiating health behaviour change $[8,14,15]$, but is less effective in goal-oriented action planning, which can lead to behaviour change relapse $[12,16]$. Not surprisingly, therefore, it has shown to be more effective and longer lasting when combined with other active treatments, rather than delivered alone [17]. Cognitive behaviour therapy (CBT) on the other hand, posits that therapeutic strategies designed to change maladaptive cognitions can lead to improvements in behaviours $[18,19]$. CBT is most commonly used to maintain behaviour change, utilising prominent strategies around relapse prevention and self-regulation [20]. In contrast to MI, CBT has shown less effectiveness in resolving ambivalence to behaviour change [21], demonstrating its greatest efficacy when working with voluntary, motivated clients [20].

Multiple studies have identified that the main factors associated with suboptimal health behaviour adoption are the lack of motivation to change and failures in strategies to maintain behaviour change [22-24]. A recent systematic review of self-regulatory mediators indicates that a lack of autonomous motivation, self-efficacy and self-regulation skills are associated with relapses in lifestyle change intervention [9]. Findings such as this, and the respective strengths and limitations of $\mathrm{MI}$ and $\mathrm{CBT}$ alone, have led to the proposal of integrating $\mathrm{MI}$ and CBT (MI-CBT) into a single intervention $[16,21,25]$. MI and CBT share components that are integral elements of both techniques [26]. Both approaches emphasise working in a collaborative, directive way with clients, with a clear focus on changing behaviour $[13,16,18,21]$. Both approaches are also understood to be most effective when focused on specific behaviours [26]. Supporting client self-efficacy and behavioural self-monitoring, key principles of MI have also been utilised in most conceptualizations of CBT [27]. It has been suggested that combined behavioural interventions offer the most effective strategy for behaviour change [28].

Several systematic reviews have demonstrated only modest effectiveness for MI alone [10, 11], and CBT alone [25] in addressing lifestyle mediators of overweight and obesity, while others have shown more promising results for behaviour change in other areas using integrated MI-CBT $[29,30]$, though the outcomes were not related to lifestyle mediators of overweight and obesity. Together these reviews provide some preliminary evidence that integrated MI-CBT might be effective for overweight and obesity. However, no systematic review has yet been undertaken that focuses specifically on the effectiveness of integrated MI-CBT interventions for addressing the lifestyle mediators of overweight and obesity in community-dwelling populations. The primary aim of this review was to examine the effectiveness of integrated MI-CBT for lifestyle mediators of overweight and obesity in community-dwelling adults. Given the prevalence of overweight and obesity, and the recommendations for lifestyle behaviour interventions, clinicians and researchers would benefit from a systematic review that focuses on identifying the benefits associated with the use of the intervention to effect lifestyle mediators of overweight and obesity.

\section{Methods}

This review with meta-analysis adheres to the guidelines outlined in the Preferred Reporting Items for Systematic Reviews and Meta-Analyses (PRISMA) Statement [31]. An electronic database search was conducted in Ovid Cochrane CENTRAL, Ovid MEDLINE, Ovid EMBASE, Ovid PsycINFO, CINAHL and Elsevier Scopus from inception until 04 October 2017. Search terms were grouped into three constructs: motivational interviewing, cognitive behaviour therapy, and health behaviour 
change. The search terms were entered as keywords or $\mathrm{MeSH}$ terms where possible, and initially searched with the OR operator; search constructs were combined using the AND operator. The complete search strategy for Embase PsycINFO is presented in detail (Additional file 1: Table S1). A manual search of reference lists from relevant articles was also conducted. Reference lists of selected trials were also examined to identify other relevant publications.

To be included in the current systematic review and meta-analysis, studies had to meet the following eligibility criteria: (1) an original, randomised controlled trial; (2) written in English-language; (3) adult population; (4) community-dwelling participants; (5) no active, serious mental health conditions, typically involving a diagnosis of psychosis; (6) intervention includes integrated MI-CBT; (7) intervention has at least one component that is delivered one-to-one; (8) outcome measures include a measured change in lifestyle mediators of overweight and obesity. Data were extracted using a standardised checklist. A unanimous decision was required between two reviewers to exclude a study during both abstract and full-text review. Where there was a lack of agreement between two reviewers, the disagreement was resolved by consensus via a third reviewer.

Data describing population characteristics, settings, intervention characteristics including duration and mode of delivery, measurement and verification of treatment fidelity, control group details, follow-up times, and outcomes were extracted from the included studies. Means and standard deviations of change scores for both intervention and control groups were included in one of the extracted studies [32]. Using these change data, the correlation coefficients were calculated for the intervention group $(r=0.50)$ and control group $(\mathrm{r}=0.50)$, with an average $r$ of 0.50 [33]. For all included studies, the standard deviation of change scores from baseline in outcome measures were calculated using a correlation coefficient of 0.5 [33], and entered directly into Review Manager 5.3 (The Nordic Cochrane Centre, The Cochrane Collaboration, Copenhagen, Denmark) for analysis [34]. Analyses based on changes from baseline were used because they are more efficient and powerful than comparison of final values, through the removal of between-person variability from the analysis [33]. Sub-group analysis was conducted to determine the potentially moderating effect of number of intervention sessions on the outcomes. All analyses were repeated for pre-post test correlations set at lower $(0.20)$ and higher $(0.80)$ values than the calculated value of 0.50 (Additional file 2: Table S2). Potential publication bias was evaluated via funnel plots (Additional file 3).

Standardized mean differences (SMD) with 95\% confidence intervals (CIs) were calculated using Review
Manager 5.3 as the mean difference divided by the pooled standard deviation [33]. For dichotomous variables, odds ratio (OR) with 95\% CIs were calculated using Review Manager 5.3. Meta-analyses were conducted on clinically homogenous data using a random effects model, to provide an estimate of the overall effect of integrated MI-CBT on health behaviour change [33]. Cohen suggests that a standardized mean difference of 0.2 is small, 0.5 is moderate, and 0.8 or more is large [35]. In keeping with recommendations, $\mathrm{I}^{2}$ was used to assess statistical heterogeneity across trials [33, 35]. Heterogeneity was considered statistically significant if the $p$-value for the Chi-square test was less than 0.10 and the $\mathrm{I}^{2}$ statistic was $50 \%$ or more $[33,36]$. In line with recommendations, if intention to treat analysis using imputed values was reported in a trial, these data were used [33]. In regards to outcome for PA, if more than one measure of PA was reported in a trial, the measure that best reflected total activity was selected and included in the analysis. Where only medians were reported, these values were treated as means and the standard deviations were derived according to the formula: standard deviation = interquartile range/1.35 [33] Study and outcome quality was assessed according to the GRADE approach for systematic reviews [33, 37]. Quality of evidence for meta-analyses began at the high level and was downgraded to lower levels of evidence when risk of bias, inconsistency, indirectness, imprecision or publication bias were present $[33,37]$.

\section{Results}

The literature search yielded a total of 1436 potentially relevant studies (Fig. 1). A total of 1241 studies were excluded after review of titles and abstracts, resulting in 195 studies undergoing full-text review. A total of 185 studies were excluded during full-text review leaving 10 studies remaining for data extraction. The characteristics of the included studies are listed in Table 1. All included studies were parallel randomised controlled trials that evaluated behaviour change relating to the lifestyle mediators of overweight and obesity. Of the included studies, 10 studies included an integrated MI-CBT intervention that measured PA as a behavioural outcome [32, 38-46], and 4 studies investigated integrated MI-CBT intervention for changes in body composition $[40-42,46]$. The outcome measures extracted from the studies related to dietary changes were highly heterogeneous and of insufficient quality to be combined for meta-analysis.

Follow-up duration varied amongst the included articles; 3 studies had a 12-month follow-up [32, 41, 44], 4 studies lasted 6 months [38, 40, 42, 43], and the remaining 3 studies had a follow-up of 4 months [46], 3 months [39], and one 1 month respectively [45]. For 


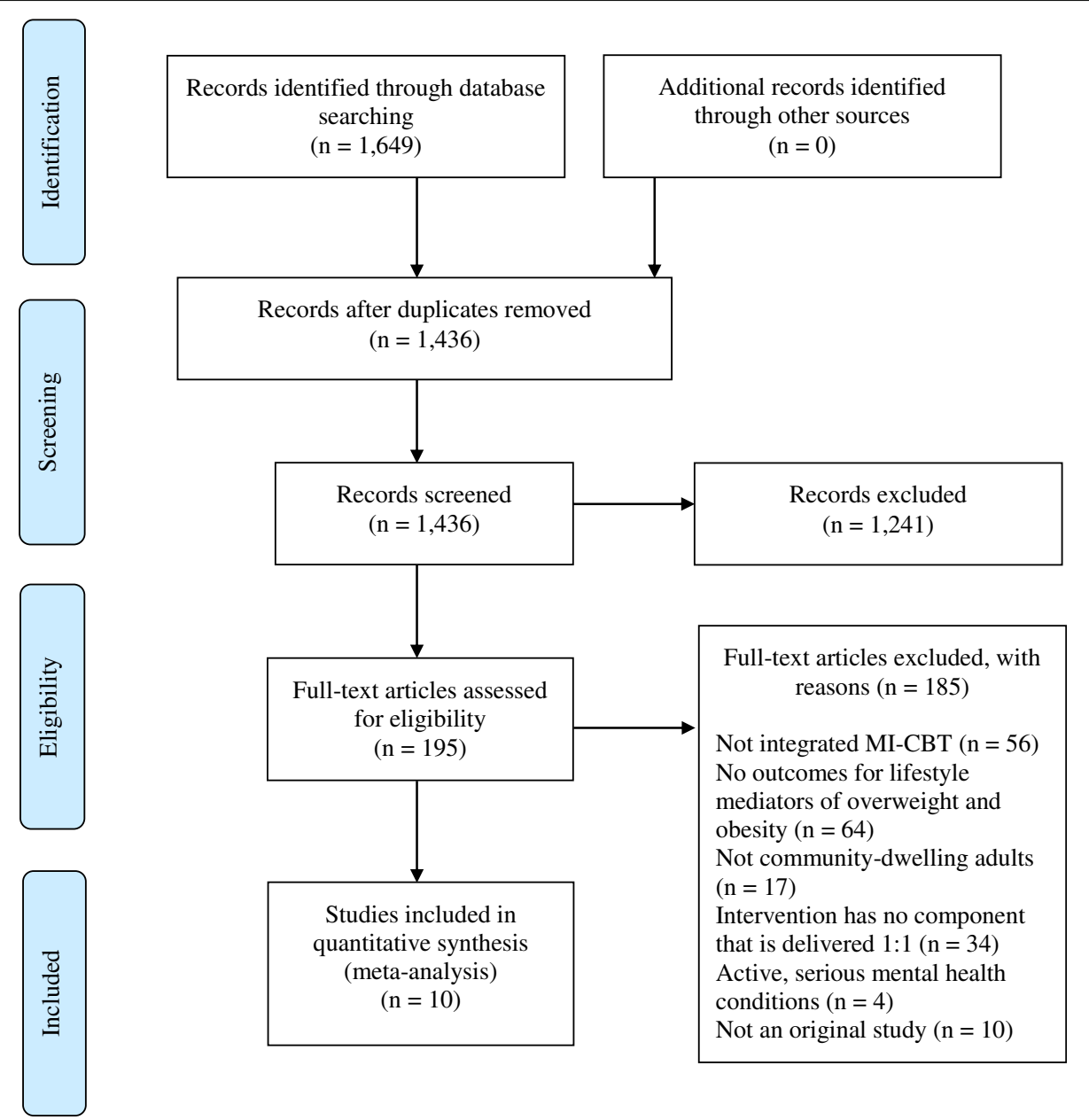

Fig. 1 Process for identification of included trials

PA outcomes, objective measures were employed in 1 study using a pedometer [41], while self-reported instruments were used in the other 9 studies [32, 38-40, 42-46]. The measures of body composition in the reviews included mass [41] and waist circumference [40, 42, 46]. Professional background of the persons delivering the intervention included PA counsellors [38], nurses [32, 41], occupational physicians [41], psychologists [42, 44], graduate students in psychology [45], health counsellors [40] and post-graduate students in sports and health science [40]. The most common methods of MI described in the studies included MI microskills (open-ended questions; affirmations; reflections; summaries) as well as feedback, affirmation and expressions of empathy. The CBT components described in the studies included problem solving, goal setting, action planning, relapse prevention, progress-related feedback and barrier identification.

The meta-analysis for MI-CBT versus standard care for change in PA demonstrated a 'moderate' quality of evidence (Additional file 4: Table S3) with a significant effect in favour of the intervention (7 studies, 1139 participants, SMD, 0.18, 95\% CI, 0.06 to 0.31, Fig. 2) [38, $39,41-44,46]$. There was a 'low' quality of evidence, with a significant effect in favour of integrated MI-CBT when the intervention lasted for 5 sessions or more (4 studies, 898 participants, SMD, 0.18, 95\% CI, 0.01 to 0.35, Fig. 2) [41-43, 46]. Interventions lasting 4 sessions or less demonstrated a 'low' quality of evidence with a non-significant effect in favour of integrated MI-CBT (3 studies, 241 participants, SMD, 0.23, 95\% CI, -0.02 to 0.49 , Fig. 2) $[38,39,44]$. The meta-analysis for integrated MI-CBT versus standard care for achieving PA guidelines demonstrated a 'low' quality of evidence (Additional file 4: Table S3) with a significant effect in favour of the intervention (4 studies, 805 participants, OR, 1.36, 95\% CI, 1.02 to 1.81 , Fig. 3) [32, 40, 43, 45]. The meta-analysis for integrated MI-CBT versus standard care for change in anthropometric measures demonstrated a 'moderate' quality of evidence (Additional file 4: Table S3) with a non-significant effect in favour of integrated MI-CBT (4 studies, 979 participants, SMD, -0.12 , 95\% CI, -0.24 to 0.01, Fig. 4) [40-42, 46]. 


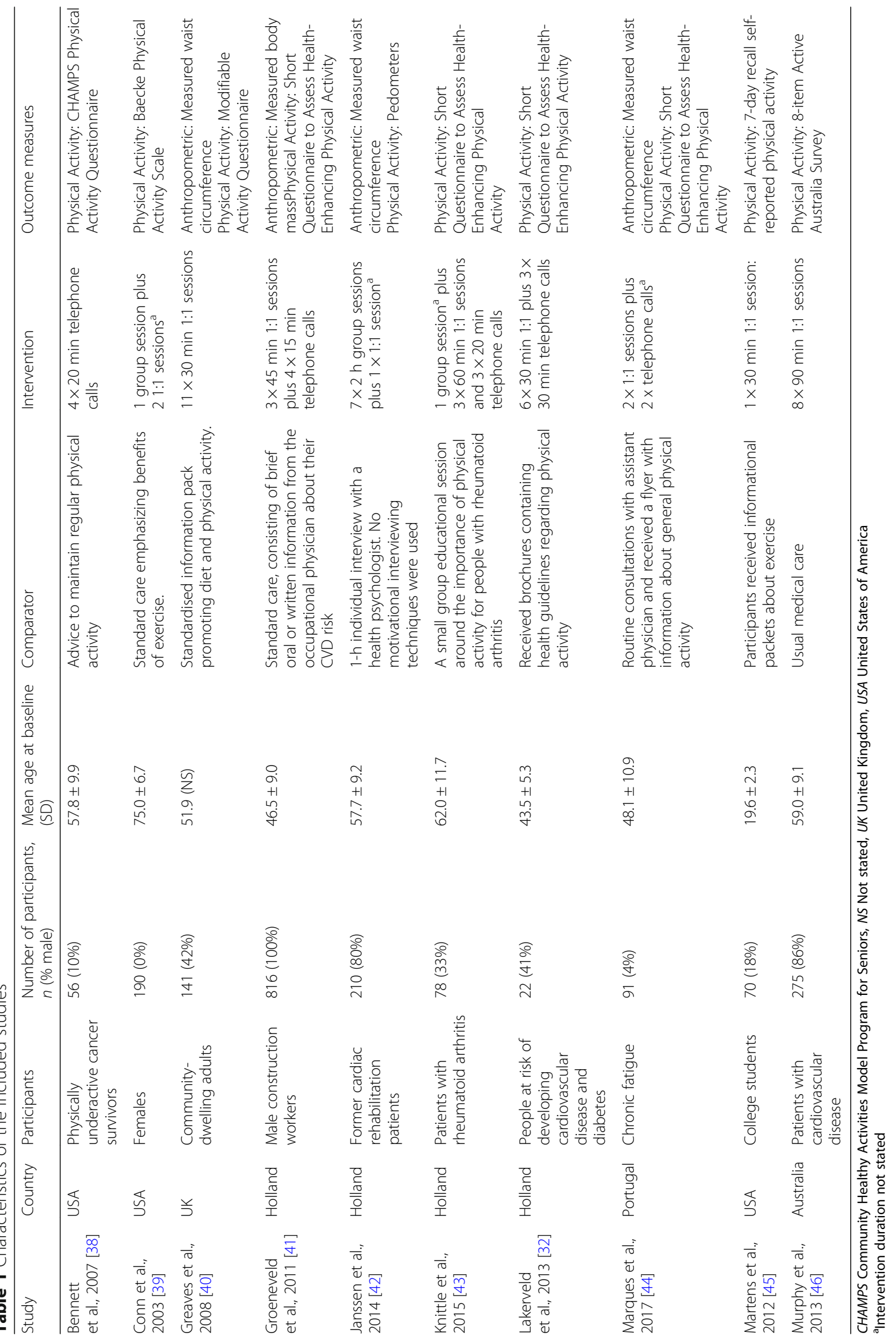




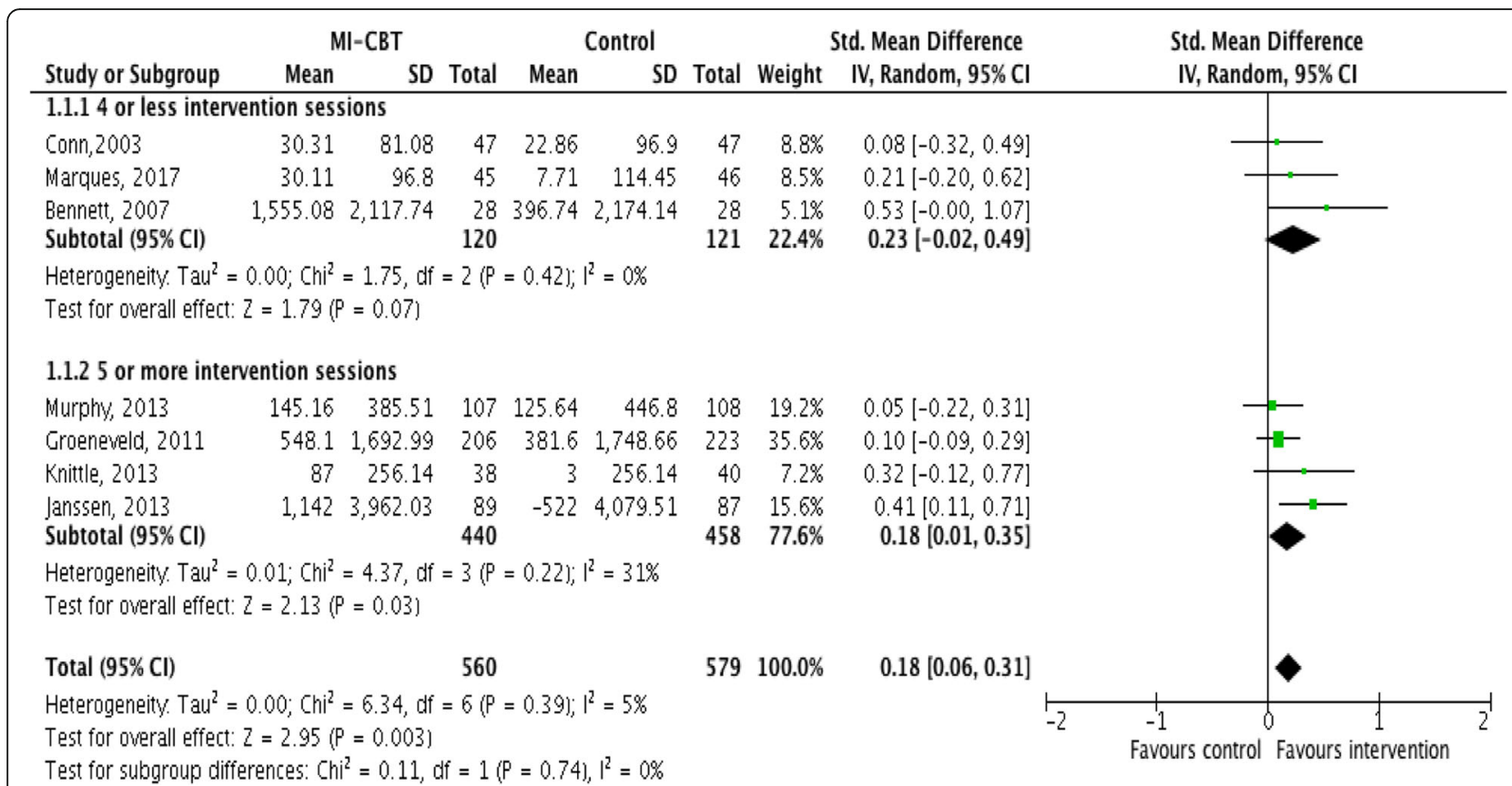

Fig. 2 Meta-analysis investigating MI-CBT for physical activity change

A sensitivity analysis of the imputed correlation coefficient revealed that effect sizes remained within the 95\% confidence interval. Funnel plots were consulted and indicated that potential publication bias could be ruled out.

\section{Discussion}

This is the first systematic review and meta-analyses to analyse the effectiveness of integrated MI-CBT for lifestyle mediators of overweight and obesity. The results provide moderate quality evidence that integrated MI-CBT has a significant, beneficial effect on PA levels, and a small, beneficial effect on body composition in community-dwelling adults. This has important implications for clinicians looking to address overweight and obesity given that even small increases in PA [47] and small changes in body composition [48] can deliver beneficial health outcomes. These findings are consistent with a previous meta-analysis reporting small but clinically significant effects for integrated MI-CBT for changes in alcohol intake [30]. Stratification of the meta-analysis for MI-CBT versus standard care for change in PA by number of intervention sessions provided low quality of evidence that interventions lasting 5 sessions or more resulted in small, significant effects on PA change (Fig. 2). Interventions lasting 4 sessions or less demonstrated a 'low' quality of evidence with a non-significant effect in favour of integrated MI-CBT (Fig. 2). This systematic search failed to yield nutritional data of sufficient quality for inclusion in the synthesis.

The results of the meta-analysis of 7 randomised controlled trials for MI-CBT versus standard care for change in PA demonstrated moderate quality of evidence with a significant effect in favour of the intervention. Incorporating exercise as a regular lifestyle behaviour is difficult for many individuals [49]. There are multiple reasons behind this, including low motivation, poor exercise tolerance, and a lack of self-efficacy and coping skills [49].

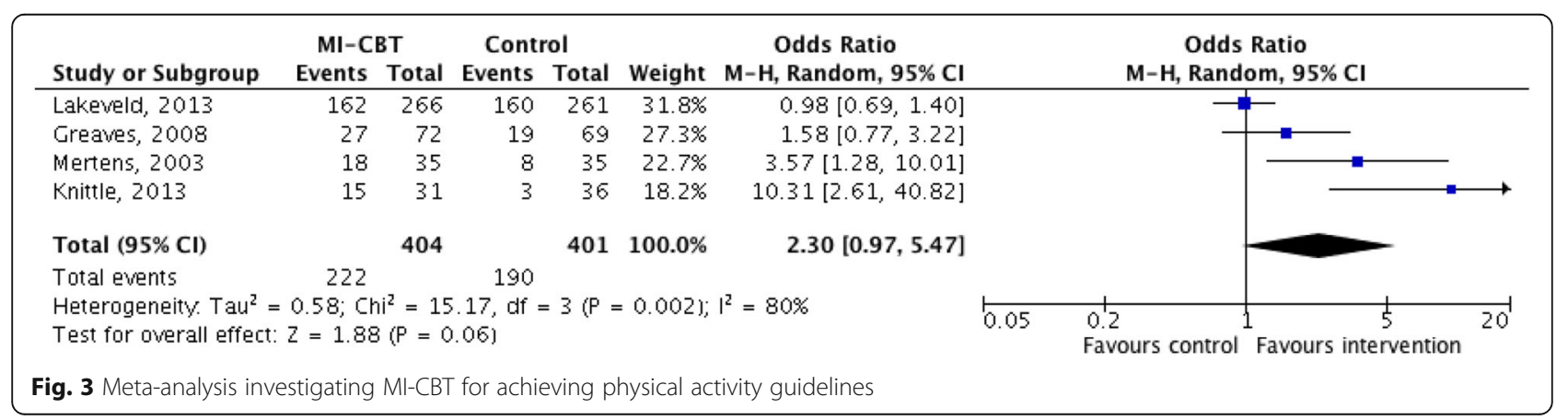




\begin{tabular}{|c|c|c|c|c|c|c|c|c|c|c|c|c|}
\hline \multirow[b]{2}{*}{ Study or Subgroup } & \multicolumn{3}{|c|}{ MI-CBT } & \multicolumn{3}{|c|}{ Control } & \multicolumn{2}{|r|}{ Std. Mean Difference } & & \multirow{2}{*}{\multicolumn{2}{|c|}{$\begin{array}{c}\text { Std. Mean Difference } \\
\text { IV, Fixed, } 95 \% \mathrm{Cl}\end{array}$}} & \\
\hline & Mean & SD & Total & Mean & SD & Total & Weight & IV, Fixed, $95 \% \mathrm{Cl}$ & & & & \\
\hline Janssen, 2013 & -2 & 10 & 89 & 0 & 11 & 87 & $17.9 \%$ & $-0.19[-0.49,0.11]$ & & 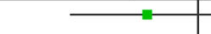 & — & \\
\hline Groeneweld, 2011 & -0.9 & 13.45 & 207 & 0.9 & 13.21 & 223 & $43.9 \%$ & $-0.13[-0.32,0.05]$ & & $\longrightarrow$ & - & \\
\hline Murphy, 2013 & -1.94 & 11.58 & 119 & -0.63 & 10.86 & 113 & $23.7 \%$ & $-0.12[-0.37,0.14]$ & & $\longrightarrow$ & - & \\
\hline Greaves, 2008 & -2 & 11.25 & 72 & -2.3 & 11.09 & 69 & $14.4 \%$ & $0.03[-0.30,0.36]$ & & & & \\
\hline Total $(95 \% \mathrm{Cl})$ & & & 487 & & & 492 & $100.0 \%$ & $-0.12[-0.24,0.01]$ & & & & \\
\hline $\begin{array}{l}\text { Heterogeneity: } \mathrm{Chi}^{2}= \\
\text { Test for owerall effect }\end{array}$ & $\begin{array}{l}0.99, \mathrm{df}= \\
Z=1.83\end{array}$ & $\begin{array}{l}=3(\mathrm{P}= \\
(\mathrm{P}=0 .\end{array}$ & $\begin{array}{l}=0.80) ; \\
07)\end{array}$ & $P^{2}=0 \%$ & & & & & -1 & $\begin{array}{c}-0.5 \\
\text { Favours MI-CBT }\end{array}$ & $\begin{array}{cc}0.5 \\
\text { Favours control }\end{array}$ & 1 \\
\hline
\end{tabular}

For individuals to positively influence obesity, they must engage regularly in PA, and maintain this behaviour over a prolonged period of time $[48,49]$. The integration of MI-CBT combines evidence informed strategies for motivation and maintenance of PA behaviour change. Of the 7 studies in this meta-analysis, only 4 had a change in PA as the primary outcome [38, 39, 43, 46]. Behaviour change interventions are most effective when they target single outcomes [10]. The lack of focus on PA change as the primary outcome in the studies included might account for the modest post-treatment effect size. In this meta-analysis, the downgrading of evidence to moderate was primarily due to a lack of sufficient methodological detail around the blinding of participants and personnel involved in the studies, resulting in an unclear risk of bias (Additional file 5: Table S4). Nevertheless, given the complexity and feasibility of blinding participants and personnel in studies using behaviour change techniques, the moderate level of evidence provides reasonably robust data supporting the use of integrated MI-CBT for PA change in community-dwelling adults.

Subgroup analysis for the effect of MI-CBT versus standard care for change in PA, stratified by number of intervention sessions demonstrated that interventions lasting 5 sessions or more resulted in a small but significant change in PA. Interventions lasting 4 sessions or less did not have a statistically significant effect on PA change. Treatment effect sizes for both MI alone [50] and CBT alone [51] have been shown to increase with higher numbers of intervention sessions, though the optimal treatment number remains unclear [50, 51]. Increased number of treatment sessions can strengthen skills around relapse prevention and self-management $[10,50,51]$, which may contribute to the clinically significant outcomes found with 5 sessions or more $[10,50]$. The broad application of behaviour change interventions in the community setting has been impeded by the lack of evidence pertaining to the optimal number of treatment sessions [52]. These meta-analyses indicate that beneficial outcomes can be derived from a small number of sessions, with increasing effect size found with 5 sessions or more.
The meta-analysis of 4 randomized controlled trials investigating MI-CBT versus standard care for achieving PA guidelines indicated that the intervention was effective at increasing PA levels in order to achieve recommended level of PA. For adults, the attainment of PA levels that approximate the recommendations for moderate activity is associated with a lower risk of mortality [53] and chronic disease [54]. In order to address overweight and obesity, the minimum of $150 \mathrm{~min} /$ week of moderate intensity exercise is required [55]. The primary measure of PA in the included studies was standard PA units, minutes per day or steps per day, for example. From this, the authors deduced the binary outcome of attaining or not attaining sufficient PA to meet the guidelines. No study in the meta-analysis investigating MI-CBT versus standard care for achieving PA guidelines provided any indication that participants were set a specific target of achieving the required minutes to meet the PA guidelines. This lack of homogeneity in study design and outcome measures in the included articles might reflect the high degree of heterogeneity found in the meta-analysis. While the meta-analysis indicates a positive effect of the intervention, the high heterogeneity and wide confidence intervals resulted in the downgrading of the quality of the evidence to low. In spite of these inconsistencies, the meta-analysis investigating MI-CBT versus standard care for achieving PA guidelines further supports the use of integrated MI-CBT for PA change. Future studies looking to measure attainment of PA guidelines should focus on clear outcome identification and uniform measurement.

The results of the meta-analysis of 4 randomized controlled trials for integrated MI-CBT versus standard care for change in anthropometric measures provided moderate quality of evidence that integrated MI-CBT has a small, positive effect on anthropometric measures (Fig. 4). Achieving long-term, sustainable changes in body composition is difficult [50]. At a minimum, the goal of obesity treatment is to prevent further weight gain [56], while minor changes in body composition are associated with decreased mortality in overweight individuals [57]. The beneficial effect on body composition demonstrated by the intervention in our analysis is 
promising. Positive change in body composition is a primary motivation for PA [58]. Positive changes in body shape have been shown to strengthen self-belief, resulting in PA maintenance [58]. All of the included studies had a 12-month follow-up, and all outcome measures were measured by research assistants. The use of objective measurement strengthens the quality of the evidence, with self-reported body weight and waist circumference measurement being prone to participant measurement error, and participant reporting bias [59]. Similar to PA, the studies included in this meta-analysis of MI-CBT versus standard care for change in anthropometric measures targeted change in multiple health behaviours, and changes in body composition outcomes was not a primary outcome in any study. When interventions target multiple health behaviours, and changes in body composition is not the primary outcome the application of behavioural change principles to body composition has been shown to decrease in priority [60]. This downgrading in perceived importance might account for the anthropometric treatment effect size found in the analysis [60].

Although the integration of MI and CBT is not a new concept [61], the confirmation and/or measurement of treatment fidelity remains difficult [61]. Fidelity scales for integrated MI-CBT have been devised and tested in the literature [61]; however none of the studies in our sample used such a fidelity measure. Therefore, the extent to which participants were actually receiving interventions is unclear, which could influence the degree of clinical homogeneity. Measurements of intervention fidelity for MI alone [62,63] and for CBT alone [64] indicate that effect size greatly increases where treatment fidelity is measured. The lack of intervention fidelity measures in the studies included in this review may be a contributory factor to the modest effect size. Of the trials included for PA change, only two trials reported measuring fidelity of the MI component $[38,40]$, with the standardised mean difference for the effect of integrated MI-CBT intervention increasing from 0.18 (95\% CI 0.06 to 0.30 ) to 0.41 ( $95 \%$ CI 0.07 to 0.75 ) when trials that did not confirm fidelity were excluded from the analysis. This effect of MI fidelity is consistent with results from previous meta-analyses which also demonstrated an increase in the standardised mean difference in trials where treatment fidelity is measured [63]. Future trials utilizing integrated MI-CBT should incorporate a measurement of fidelity into the study design.

\section{Strengths}

This is the first systematic review and meta-analysis undertaken that provides evidence to support the use of integrated MI-CBT for changes in PA and body composition in community-dwelling adults. While the demonstrated effect was modest, the combination of MI and
CBT is potentially advantageous for a number of reasons. With evidence suggesting that even small increases in PA and body composition change can deliver positive health benefits, a modest effect size, as demonstrated in this review, is likely to deliver important health outcomes [48]. The integration of MI-CBT might overcome the documented shortcomings in both interventions delivered alone, while maintaining a collaborative, directive approach [16]. While interventions incorporating behavioural or psychological components have demonstrated modest efficacy for lifestyle mediators of overweight and obesity overall, these interventions do not result in adverse effects, and generally lead to improvements in psychological well-being [65]. As single intervention studies have indicated that larger post-treatment effect sizes are produced if MI and CBT are delivered with fidelity $[50,51]$, it can be hypothesised that integrated MI-CBT interventions adhering to higher rates of fidelity have the potential to produce increased effect sizes [61]. Another potential advantage to using integrated MI-CBT for addressing lifestyle mediators of overweight and obesity lies in the range of health professionals that were able to deliver the intervention. This clinical diversity might be advantageous when applying the intervention across multiple sectors of the community-dwelling population, especially given the previously mentioned prevalence of overweight and obesity. For clinical interest and uptake, as indicated by the findings of these meta-analyses, higher quality randomised controlled trials with detailed interventions, extended follow-up periods, and measures of treatment fidelity are required $[50,51]$.

\section{Limitations}

There are a number of limitations of our review that need to be considered. The number of included trials was restricted by the use of the rigid search criteria designed to assess the combined effects of MI-CBT on lifestyle mediators of overweight and obesity. For example, it was not possible to extract sufficient data to undertake meta-analyses for dietary changes. Excluding languages other than English might also introduce a bias and reduce the precision of estimated treatment effects. Secondly, self-reported tools were used to measure changes in PA change in 9 of the 10 included trials. This lack of an objectively measured outcomes resulted in a higher risk of bias [59]. Thirdly, this review and meta-analyses included a number of small trials, undertaken on restrictive populations, which might have influenced the observed effect sizes [33]. Finally, there may have been an impact on external validity from combining data from studies on participants with diverse health conditions. Nevertheless, for all but one of the meta-analyses, heterogeneity was low, and research 
continually indicates that increasing PA and positive body composition changes have favourable health effects for the majority of the population [47].

\section{Conclusions}

Despite the small number of high quality randomised trials, this analysis indicates that integrated MI-CBT leads to modest improvements in PA and body composition changes amongst community-dwelling adults.

The emerging evidence to support the use of MI-CBT interventions for promoting the adoption and maintenance of health behaviour change has potential importance in addressing the high rates of obesity. The intervention can be delivered by a range of health professionals and can be incorporated readily into clinical practice. In order to make stronger recommendations regarding the effectiveness of this intervention for lifestyle mediators of overweight and obesity, more high quality randomised controlled trials are required. Such studies should include sufficient follow-up durations to determine the long-term effects of the interventions. Finally, trials should also include clearly defined interventions, objective outcome measures and assessments of treatment fidelity.

\section{Additional files}

Additional file 1: Table S1. Search strategy and results: Ovid PsycINFO. (DOCX $44 \mathrm{~kb}$ )

Additional file 2: Table S2. Sensitivity analyses of imputed correlation coefficients for meta-analyses investigating MI-CBT for physical activity change and anthropometric change. (DOCX $12 \mathrm{~kb}$ )

Additional file 3: Funnel plots of meta-analyses investigating MI-CBT for physical activity change and anthropometry change. (DOCX 2897 kb)

Additional file 4: Table S3. Quality of evidence of Integrated Motivational Interviewing and Cognitive-Behaviour Therapy compared to standard care for physical activity change and anthropometric change. (DOCX 19 kb)

Additional file 5: Table 4. Risk of bias for included studies. (DOCX $13 \mathrm{~kb}$ )

\section{Abbreviations}

BMI: Body Mass Index; CBT: Cognitive Behaviour Therapy; Cl: Confidence Interval; Ml: Motivational Interviewing; MI-CBT: Integrated Ml and CBT; PA: Physical Activity; SMD: Standardized Mean Differences

\section{Acknowledgements}

The authors would like to thank Dr. Karl Landorf from La Trobe University, Melbourne who provided advice on the manuscript.

\section{Availability of data and materials}

The dataset used and analysed during the current study are available from the corresponding author on reasonable request.

\section{Authors' contributions}

All authors conceived the idea, contributed to the search strategy employed. $\mathrm{SB}^{1}$ conducted the literature search, quality assessment, data extraction and analysis, and wrote the draft manuscript. All authors contributed to the interpretation of the results and critically reviewed the manuscript. All authors read and approved the final manuscript.
Ethics approval and consent to participate

Not applicable.

\section{Consent for publication}

Not applicable.

\section{Competing interests}

The authors declare they have no competing interests.

\section{Publisher's Note}

Springer Nature remains neutral with regard to jurisdictional claims in published maps and institutional affiliations.

\section{Author details}

'La Trobe University, La Trobe Rural Health School, PO Box 199, Bendigo, VIC 3552, Australia. 'La Trobe University, School of Psychology and Public Health, Bundoora, VIC 3068, Australia.

Received: 30 June 2018 Accepted: 24 September 2018

Published online: 05 October 2018

\section{References}

1. Flegal KM, Carroll MD, Ogden CL, Curtin LR. Prevalence and trends in obesity among US adults, 1999-2008. Jama. 2010;303(3):235-41.

2. Organization WH: World Health Organization obesity and overweight fact sheet. 2016.

3. Malnick SD, Knobler H. The medical complications of obesity. Qjm. 2006; 99(9):565-79.

4. Must A, Spadano J, Coakley EH, Field AE, Colditz G, Dietz WH. The disease burden associated with overweight and obesity. Jama. 1999;282(16):1523-9.

5. Stevens GA, Singh GM, Lu Y, Danaei G, Lin JK, Finucane MM, Bahalim AN, McIntire RK, Gutierrez HR, Cowan M, et al. National, regional, and global trends in adult overweight and obesity prevalences. Popul Health Metrics. 2012;10:22.

6. Hruby A, Hu FB. The epidemiology of obesity: a big picture. Pharmacoeconomics. 2015;33(7):673-89.

7. Pigeyre M, Rousseaux J, Trouiller P, Dumont J, Goumidi L, Bonte D, Dumont MP, Chmielewski A, Duhamel A, Amouyel P, et al. How obesity relates to socio-economic status: identification of eating behavior mediators. Int J Obes. 2016:40(11):1794-801.

8. Looney SM, Raynor HA. Behavioral lifestyle intervention in the treatment of obesity. Health Serv Insights. 2013;6:15-31.

9. Teixeira PJ, Carraca EV, Marques MM, Rutter H, Oppert JM, De Bourdeaudhuij I, Lakerveld J, Brug J. Successful behavior change in obesity interventions in adults: a systematic review of self-regulation mediators. BMC Med. 2015;13:84.

10. Armstrong MJ, Mottershead TA, Ronksley PE, Sigal RJ, Campbell TS, Hemmelgarn BR. Motivational interviewing to improve weight loss in overweight and/or obese patients: a systematic review and meta-analysis of randomized controlled trials. Obes Rev. 2011;12(9):709-23.

11. Donnelly JE, Blair SN, Jakicic JM, Manore MM, Rankin JW, Smith BK. American College of Sports Medicine position stand. Appropriate physical activity intervention strategies for weight loss and prevention of weight regain for adults. Med Sci Sports Exerc. 2009:41(2):459-71.

12. Lundahl B, Burke BL. The effectiveness and applicability of motivational interviewing: a practice-friendly review of four meta-analyses. J Clin Psychol. 2009;65(11):1232-45

13. Miller WR, Rollnick S. Motivational interviewing: Helping people change (applications of motivational interviewing). New York: The Guilford Press; 2012.

14. Rubak S, Sandbæk A, Lauritzen T, Christensen B. Motivational interviewing: a systematic review and meta-analysis. Br J Gen Pract. 2005;55(513):305-12.

15. Heckman CJ, Egleston BL, Hofmann MT. Efficacy of motivational interviewing for smoking cessation: a systematic review and meta-analysis Tob Control. 2010;19(5):410-6.

16. Naar-King S, Earnshaw P, Breckon J. Towards a universal maintenance intervention: Integrating motivational interviewing with cognitive-behavioral strategies for maintenance of behavior change, vol. 27; 2013.

17. Hettema J, Steele J, Miller WR. Motivational interviewing. Annu Rev Clin Psychol. 2005;1:91-111. 
18. Beck AT. Cognitive therapy: nature and relation to behavior therapy republished article. Behav Ther. 2016;47(6):776-84.

19. Ellis A: Reason and emotion in Psychotherapy. 1962.

20. Ryan RM, Lynch MF, Vansteenkiste M, Deci EL. Motivation and autonomy in counseling, psychotherapy, and behavior change: a look at theory and. Practice 147. Couns Psychol. 2011;39(2):193-260.

21. Westra H. Managing resistance in cognitive behavioural therapy: the application of motivational interviewing in mixed anxiety and depression. Cogn Behav Ther. 2004;33(4):161-75.

22. Ashenden R, Silagy C, Weller D. A systematic review of the effectiveness of promoting lifestyle change in general practice. Fam Pract. 1997;14(2):160-76.

23. Piasecki TM. Relapse to smoking. Clin Psychol Rev. 2006;26(2):196-215.

24. Wing RR, Phelan S. Long-term weight loss maintenance. Am J Clin Nutr. 2005;82(1):222S-5S.

25. Burke BL. What can motivational interviewing do for you? Cogn Behav Pract. 2011;18(1):74-81

26. Flynn HA. Setting the stage for the integration of motivational interviewing with cognitive behavioral therapy in the treatment of depression. Cogn Behav Pract. 2011;18(1):46-54.

27. O'Leary KD, Wilson GT: Behavior therapy: application and outcome: prentice-hall, Inc; 1987.

28. Burgess $E$, Hassmen P, Welvaert M, Pumpa KL. Behavioural treatment strategies improve adherence to lifestyle intervention programmes in adults with obesity: a systematic review and meta-analysis. Clinical obesity. 2017;7(2):105-14.

29. Baker AL, Thornton LK, Hiles S, Hides L, Lubman DI. Psychological interventions for alcohol misuse among people with co-occurring depression or anxiety disorders: a systematic review. J Affect Disord. 2012;139(3):217-29.

30. Riper H, Andersson G, Hunter SB, Wit J, Berking M, Cuijpers P. Treatment of comorbid alcohol use disorders and depression with cognitive-behavioural therapy and motivational interviewing: a meta-analysis. Addiction. 2014; 109(3):394-406.

31. Moher D, Liberati A, Tetzlaff J, Altman DG, Group P. Preferred reporting items for systematic reviews and meta-analyses: the PRISMA statement. PLoS Med. 2009;6(7):e1000097.

32. Lakerveld J, Bot SD, Chinapaw MJ, van Tulder MW, Kostense PJ, Dekker JM, Nijpels G. Motivational interviewing and problem solving treatment to reduce type 2 diabetes and cardiovascular disease risk in real life: a randomized controlled trial. Int J Behav Nutr Phys Act. 2013;10(1):47.

33. Higgins JP, Green S: Cochrane handbook for systematic reviews of interventions, vol. 4. Chichester: John Wiley; 2011.

34. Collaboration C. Review manager (RevMan) version 5.3. The Nordic Cochrane Centre: Copenhagen; 2014

35. Cohen J. Statistical power analysis for the behavioral. Sciences 2 nd edn. Hillsdale: Erlbaum Associates; 1988.

36. Thorlund K, Imberger G, Johnston BC, Walsh M, Awad T, Thabane L, Gluud C, Devereaux P, Wetterslev J. Evolution of heterogeneity (I2) estimates and their 95\% confidence intervals in large meta-analyses. PLoS One. 2012;7(7): e39471.

37. Balshem $H$, Helfand $M$, Schünemann HJ, Oxman AD, Kunz R, Brozek J, Vist GE, Falck-Ytter Y, Meerpohl J, Norris S. GRADE guidelines: 3. Rating the quality of evidence. J Clin Epidemiol. 2011;64(4):401-6.

38. Bennett JA, Lyons KS, Winters-Stone K, Nail LM, Scherer J. Motivational interviewing to increase physical activity in long-term cancer survivors: a randomized controlled trial. Nurs Res. 2007;56(1):18-27.

39. Conn VS, Burks KJ, Minor MA, Mehr DR. Randomized trial of 2 interventions to increase older women's exercise. Am J Health Behav. 2003;27(4):380-8.

40. Greaves CJ, Middlebrooke A, O'Loughlin L, Holland S, Piper J, Steele A, Gale T, Hammerton F, Daly M. Motivational interviewing for modifying diabetes risk: a randomised controlled trial. Br J Gen Pract. 2008;58(553):535-40.

41. Groeneveld IF, Proper KI, van der Beek AJ, Hildebrandt VH, van Mechelen W. Short and long term effects of a lifestyle intervention for construction workers at risk for cardiovascular disease: a randomized controlled trial. BMC Public Health. 2011;11(1):836.

42. Janssen V, De Gucht V, van Exel H, Maes S. A self-regulation lifestyle program for post-cardiac rehabilitation patients has long-term effects on exercise adherence. J Behav Med. 2014;37(2):308-21.

43. Knittle K, De Gucht V, Hurkmans E, Peeters A, Ronday K, Maes S, Vlieland TV. Targeting motivation and self-regulation to increase physical activity among patients with rheumatoid arthritis: a randomised controlled trial. Clin Rheumatol. 2015;34(2):231-8.
44. Marques MM, de Gucht V, Leal I, Maes S. Efficacy of a randomized controlled self-regulation based physical activity intervention for chronic fatigue: mediation effects of physical activity progress and selfregulation skills. J Psychosom Res. 2017;94:24-31.

45. Martens MP, Buscemi J, Smith AE, Murphy JG. The short-term efficacy of a brief motivational intervention designed to increase physical activity among college students. J Phys Act Health. 2012;9(4):525-32

46. Murphy BM, Worcester MU, Higgins RO, Elliott PC, Le Grande MR, Mitchell F, Navaratnam H, Turner A, Grigg L, Tatoulis J. Reduction in 2-year recurrent risk score and improved behavioral outcomes after participation in the "beating heart problems" self-management program: results of a randomized controlled trial. J Cardiopulm Rehabil Prev. 2013;33(4):220-8.

47. Sattelmair J, Pertman J, Ding EL, Kohl HW, Haskell W, Lee I-M: Dose response between physical activity and risk of coronary heart disease: a meta-analysis. Circulation 2011:CIRCULATIONAHA. 110.010710.

48. Magkos F, Fraterrigo G, Yoshino J, Luecking C, Kirbach K, Kelly SC, de las Fuentes L, He S, Okunade AL, Patterson BW. Effects of moderate and subsequent progressive weight loss on metabolic function and adipose tissue biology in humans with obesity. Cell Metab. 2016;23(4):591-601.

49. Dalle Grave R, Calugi S, Centis E, El Ghoch M, Marchesini G. Cognitivebehavioral strategies to increase the adherence to exercise in the management of obesity. J Obes. 2011;2010.

50. Lundahl BW, Kunz C, Brownell C, Tollefson D, Burke BL. A meta-analysis of motivational interviewing: twenty-five years of empirical studies. Res Soc Work Pract. 2010;20(2):137-60.

51. Hofmann SG, Asnaani A, Vonk IJ, Sawyer AT, Fang A. The efficacy of cognitive behavioral therapy: a review of meta-analyses. Cogn Ther Res. 2012;36(5):427-40.

52. Landry A, Madson M, Thomson J, Zoellner J, Connell C, Yadrick K. A randomized trial using motivational interviewing for maintenance of blood pressure improvements in a community-engaged lifestyle intervention: HUB city steps. Health Educ Res. 2015;30(6):910-22.

53. Leitzmann MF, Park Y, Blair A, Ballard-Barbash R, Mouw T, Hollenbeck AR, Schatzkin A. Physical activity recommendations and decreased risk of mortality. Arch Intern Med. 2007;167(22):2453-60.

54. Sesso HD, Paffenbarger RS, Lee I-M. Physical activity and coronary heart disease in men: the Harvard alumni health study. Circulation. 2000;102(9):975-80.

55. Jakicic JM, Clark K, Coleman E, Donnelly JE, Foreyt J, Melanson E, Volek J, Volpe SL. Appropriate intervention strategies for weight loss and prevention of weight regain for adults. Med Sci Sports Exerc. 2001.

56. Sharma AM, Padwal R. Obesity is a sign-over-eating is a symptom: an aetiological framework for the assessment and management of obesity. Obes Rev. 2010;11(5):362-70.

57. Williamson DF, Thompson TJ, Thun M, Flanders D, Pamuk E, Byers T. Intentional weight loss and mortality among overweight individuals with diabetes. Diabetes Care. 2000;23(10):1499-504.

58. Kim S-J, Cho B-H. The effects of empowered motivation on exercise adherence and physical fitness in college women. J Exerc Rehabil. 2013;9(2):278.

59. Stommel M, Schoenborn CA. Accuracy and usefulness of BMI measures based on self-reported weight and height: findings from the NHANES \& NHIS 2001-2006. BMC Public Health. 2009;9(1):421.

60. Prochaska JJ, Prochaska JO. A review of multiple health behavior change interventions for primary prevention. Am J Lifestyle Med. 2011;5(3):208-21.

61. Haddock G, Beardmore R, Earnshaw P, Fitzsimmons M, Nothard S, Butler R, Eisner E, Barrowclough C. Assessing fidelity to integrated motivational interviewing and CBT therapy for psychosis and substance use: the MI-CBT fidelity scale (MI-CTS). J Ment Health. 2012;21(1):38-48.

62. Moyers TB, Martin T, Manuel JK, Hendrickson SM, Miller WR. Assessing competence in the use of motivational interviewing. J Subst Abus Treat. 2005;28(1):19-26.

63. O'Halloran PD, Blackstock F, Shields N, Holland A, lles R, Kingsley M, Bernhardt J, Lannin N, Morris ME, Taylor NF. Motivational interviewing to increase physical activity in people with chronic health conditions: a systematic review and meta-analysis. Clin Rehabil. 2014;28(12):1159-71.

64. Bellg AJ, Borrelli B, Resnick B, Hecht J, Minicucci DS, Ory M, Ogedegbe G, Orwig D, Ernst D, Czajkowski S. Enhancing treatment fidelity in health behavior change studies: best practices and recommendations from the $\mathrm{NIH}$ behavior change consortium. Health Psychol. 2004;23(5):443.

65. Lasikiewicz N, Myrissa K, Hoyland A, Lawton C. Psychological benefits of weight loss following behavioural and/or dietary weight loss interventions. A systematic research review. Appetite. 2014;72:123-37. 